\title{
ECM1 modified HF-MSCs targeting HSC attenuate liver cirrhosis by inhibiting the TGF- $\beta /$ Smad signaling pathway
}

\author{
Qi Liu ${ }^{1}$, Chengqian Lv ${ }^{1}$, Qianqian Huang ${ }^{1}$, Lei Zhao ${ }^{1}$, Xiaoli Sun ${ }^{1}$, Dandan Ning ${ }^{1}$, Jingyang Liu ${ }^{1}$, Yanan Jiang ${ }^{2,3}$ and Shizhu Jin ${ }^{1}{ }^{\circledR}$
}

(c) The Author(s) 2022

Hair follicle-derived mesenchymal stem cells (HF-MSCs) show considerable therapeutic potential for liver cirrhosis (LC). To improve the effectiveness of naïve HF-MSC treatments on LC, we used bioinformatic tools to identify an exogenous gene targeting HSCs among the differentially expressed genes (DEGs) in LC to modify HF-MSCs. Extracellular matrix protein 1 (ECM1) was identified as a DEG that was significantly downregulated in the cirrhotic liver. Then, ECM1-overexpressing HF-MSCs (ECM1-HF-MSCs) were transplanted into mice with LC to explore the effectiveness and correlated mechanism of geneoverexpressing HF-MSCs on LC. The results showed that ECM1-HF-MSCs significantly improved liver function and liver pathological injury in LC after cell therapy relative to the other treatment groups. Moreover, we found that ECM1-HF-MSCs homed to the injured liver and expressed the hepatocyte-specific surface markers ALB, CK18, and AFP. In addition, hepatic stellate cell (HSC) activation was significantly inhibited in the cell treatment groups in vivo and in vitro, especially in the ECM1HF-MSC group. Additionally, TGF- $\beta / S$ mad signal inhibition was the most significant in the ECM1-HF-MSC group in vivo and in vitro. The findings indicate that the genetic modification of HF-MSCs with bioinformatic tools may provide a broad perspective for precision treatment of LC.

Cell Death Discovery (2022)8:51 ; https://doi.org/10.1038/s41420-022-00846-4

\section{INTRODUCTION}

Liver cirrhosis (LC) is induced by a variety of causes, including infectious factors, chemical injury, and metabolic and genetic factors. There is no effective way to cure LC except for orthotopic liver transplantation (OLT). However, most patients with cirrhosis do not have the opportunity to receive OLT due to a lack of financial resources, immune rejection, or shortage of donor livers, among other reasons [1-4]. Considering that the excessive deposition of extracellular matrix (ECM) caused by hepatic stellate cell (HSC) activation is a critical aspect in the course of LC development [5-8], more effective strategies targeting HSCs to treat LC are urgently needed.

The emerging application of mesenchymal stem cells (MSCs) provides a promising method for treating end-stage hepatic disease. Hair follicle mesenchymal stem cells (HF-MSCs) are derived from the bulge of hair follicles [9] and present broad application prospects considering their characteristics of rich source materials, easy access, low immunogenicity, and almost no limitation according to age [10]. In addition, the extraction of HF-MSCs is not as traumatic as bone marrow-derived mesenchymal stem cells (BM-MSCs), and more importantly, HF-MSCs show greater proliferation than BM-MSCs [11]. HF-MSCs have been proven to have the ability to differentiate into tissuespecific cells such as fat, bone, cartilage, smooth muscle cells, neurogliocytes, melanocytes, and hepatocytes [12-15], which indicates that HF-MSCs may have extensive prospects for liver disease treatment. Despite the above advantages, the role of naïve MSCs in LC treatment remains to be improved [16, 17], and the overexpression of genes in MSCs has proven to be a more effective way to treat liver fibrosis/cirrhosis [18-22].

High-throughput sequencing and gene chips provide the ability to elucidate the changes in genetic information during the development of liver diseases. Here, we used bioinformatic tools to identify an HSC-related target gene among the differentially expressed genes (DEGs) in LC.

Extracellular matrix protein 1 (ECM1) was identified as a DEG downregulated in LC for the modification of HF-MSCs. ECM1 is a secreted glycoprotein and is involved in embryonic chondrogenesis, skin differentiation, angiogenesis, and cell proliferation [23-27]. ECM1 is mainly secreted by hepatocytes and contributes to the maintenance of hepatic homeostasis. The amount of ECM1 produced by hepatocytes is reduced when liver fibrosis occurs, while exogenous ECM1 supplementation can reverse hepatofibrosis by blocking HSC activation [28]. Therefore, increasing the level of ECM1 continuously and steadily in the damaged liver may become a new strategy to reverse LC.

In our study, we applied ECM1-overexpressing HF-MSCs in LC to evaluate the effectiveness and mechanism of genetransfected HF-MSCs, which may provide a relevant theoretical basis for the application of ECM1-HF-MSCs in LC treatment.

\footnotetext{
${ }^{1}$ Department of Gastroenterology and Hepatology, The Second Affiliated Hospital of Harbin Medical University, Harbin, Heilongjiang Province 150086, P. R. China. ${ }^{2}$ Department of Pharmacology (State-Province Key Laboratories of Biomedicine-Pharmaceutics of China, Key Laboratory of Cardiovascular Research, Ministry of Education), College of Pharmacy of Harbin Medical University, Harbin, Heilongjiang Province 150081, P. R. China. ${ }^{3}$ Translational Medicine Research and Cooperation Center of Northern China, Heilongjiang Academy of Medical Sciences, Harbin, Heilongjiang Province 150086, P. R. China. ${ }^{{ }^{-}}$email: drshizhujin@hrbmu.edu.cn
}

Received: 7 December 2021 Revised: 5 January 2022 Accepted: 20 January 2022

Published online: 08 February 2022 


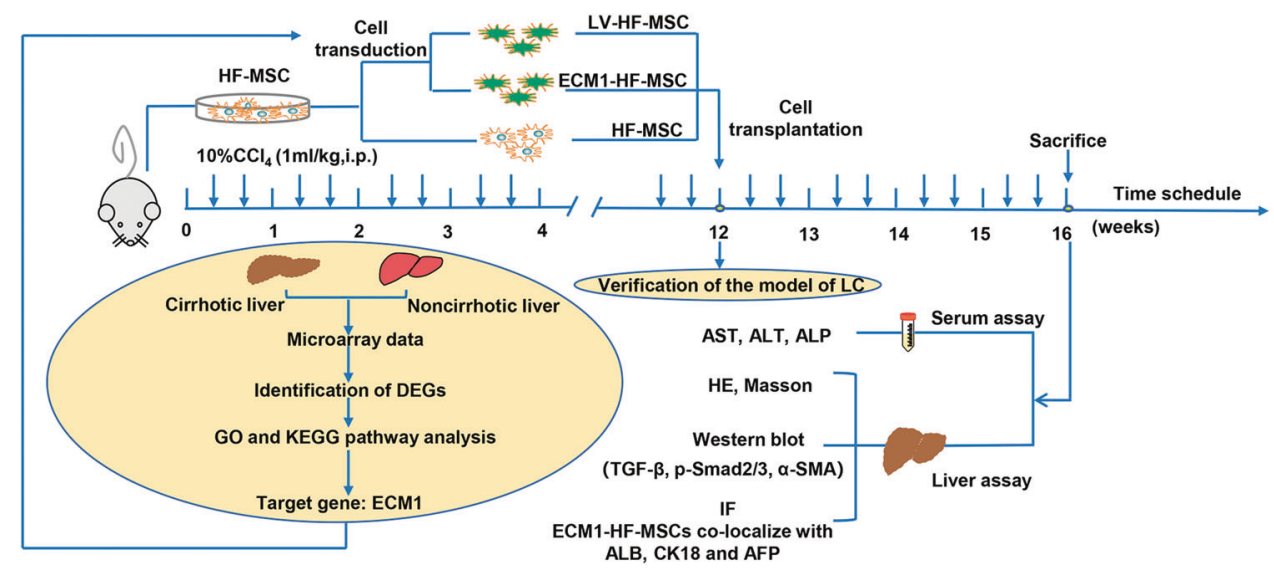

Fig. 1 Overview of the experimental design. The DEGs related to LC were analyzed using bioinformatic tools. ECM1 was selected from the DEGs and was then used to modify HF-MSCs. The cells were injected into mice with $\mathrm{CCl}_{4}$-induced LC through the tail vein. Four weeks after cell transplantation, serum and liver tissue were collected for HE and Masson staining and Western blotting and immunofluorescence detection.

\section{MATERIALS AND METHODS \\ Identification of DEGs from microarray data}

The whole experimental design is shown in Fig. 1. The gene expression profile GSE103580 dataset was downloaded from the GEO database (http://www.ncbi.nlm.nih.gov/geo). The GSE103580 dataset contains 86 samples, including 67 cirrhotic samples and 19 noncirrhotic samples. The online analysis software GEO2R (http://www.ncbi.nlm.nih.gov/geo/ geo2r) was used to analyze the DEGs between the cirrhotic group and the noncirrhotic group, according to a screening threshold of $P<0.05$ and a fold change $\geq 1.5$. Volcano plots and heat maps generated by the $R$ language packages ggpubr and pheatmap were used to display the screened DEGs.

\section{Gene ontology (GO) and kyoto encyclopedia of genes and genomes (KEGG) pathway enrichment analysis of DEGs} To analyze the function of DEGs in LC, GO analysis and KEGG pathway enrichment analysis of DEGs were performed using the online Database for Annotation, Visualization, and Integrated Discovery (DAVID, version 6.8; david.ncifcrf.gov) tool, and the enrichment difference was regarded as significant when $p<0.05$. The analysis results are shown in bubble charts using the $\mathrm{R}$ language package ggplot.

\section{Protein-protein interaction network of DEGs encoding proteins}

To visualize the proteins encoded by DEGs, the STRING database (http://string$\mathrm{db}$. org; version 10.0) was used to construct a PPI network with a confidence of 0.40. Then, the filtered PPI results were imported into Cytoscape 3.7.2 software to construct a visual molecular interaction network.

\section{Experimental animals}

C57BL/6J (specific pathogen-free) male mice were used in this experiment. Seven- to nine-day-old mice were chosen to extract primary HF-MSCs, and sixto seven-week-old mice weighing $18-22 \mathrm{~g}$ were selected for the subsequent experiments. The mice were all from the scientific research center of the Second Affiliated Hospital of Harbin Medical University. The mice could obtain feed without intervention at the scientific research center. All animal experimental protocols were conducted in accordance with the principles of medical ethics and approved by the Ethics Committee of the Second Affiliated Hospital of Harbin Medical University (NO. SYDW2019-240).

Our pre-experimental results show that the difference was statistically significant when the minimum sample size was 6 . Mice that died during the experiment were excluded from the experiment, and the surviving mice were used in subsequent experiments. No blinding was done due to the obvious grouping basis by experimenter.

\section{Isolation and culture of primary HF-MSCs}

Healthy mice were anesthetized, and the skin around their beard was disinfected with medical alcohol. Then, the skin around the beard was resected and minced into soybean-sized pieces. Type I collagenase $(0.1 \%$,
Sigma-Aldrich, St Louis, MO, USA) was used to hydrolyze the collagen components of connective tissue at $4^{\circ} \mathrm{C}$. After $13 \mathrm{~h}$, the digestion reaction was stopped by the addition of fetal bovine serum (FBS, ScienCell, USA), and the collagenase attached to the tissues was removed with PBS. The hair follicles were peeled off with microtweezers under a binocular microscope within one hour. Then, one hair follicle was inoculated in a cell culture plate containing DMEM/F12 (Gibco, Gaithersburg, MD, USA), 15\% FBS (ScienCell), and $1 \%$ penicillin-streptomycin (Gibco). Cells were passaged when they reached $70-80 \%$ confluence approximately 12-14 days later. Second- or third-generation HF-MSCs with a good growth status were used for subsequent experiments. All operations were performed in a sterile environment.

\section{Identification of HF-MSCs}

Third-generation HF-MSCs were used for phenotyping analysis. The cells $\left(1 \times 10^{6}\right.$ cells per specimen) were incubated with FITC-, PE-, PerCP-, and APC-labeled monoclonal antibodies against CD29, CD90, CD43, and CD31 for half an hour at $4{ }^{\circ} \mathrm{C}$ without light. The antibodies were then washed off with PBS. The phenotypes were analyzed by a FACSCanto II flow cytometer (BD Biosciences, USA).

The multi-differentiation potential of the HF-MSCs was determined by assessing their ability to differentiate into adipocytes and osteocytes. Alizarin red (Sigma-Aldrich) staining was used to detect mineralized nodules in osteogenic differentiated cells, and the lipid droplets in the differentiated cells were detected by Oil red O (Sigma-Aldrich) staining after adipogenic induction according to the instructions.

\section{Lentivirus transfection of HF-MSCs}

Lentiviral vectors (LVs) were used to carry the target gene ECM1 to infect HF-MSCs to achieve lasting expression of ECM1. LVs (GOSL0219611) were purchased from Shanghai Gene Chemical Company, including one blank LV encoding only green fluorescent protein (GFP) (GFP-LV) and one ECM1overexpressing LV encoding both GFP and the ECM1 protein (ECM1-LV). The blank LV was used for the preliminary experiment to achieve the optimal multiplicity of infection (MOI). Third-generation HF-MSCs were inoculated into 96 -well plates for $24 \mathrm{~h}$ until the cells filled $30 \%$ of each well. The HF-MSCs were then infected with GFP-LV (LV-HF-MSCs) at different MOls $(20,40,60,80$, and 100). After $48 \mathrm{~h}$, the fluorescence intensity and transfection efficiency were detected to obtain the optimal MOI. Then, the HF-MSCs were transfected with ECM1-LV at the optimal MOI for $72 \mathrm{~h}$ for subsequent experiments.

\section{Animal model of LC and experimental design}

To induce the mouse $\mathrm{LC}$ model, $10 \%$ carbon tetrachloride $\left(\mathrm{CCl}_{4}\right.$, diluted in oil) was injected into the abdominal cavity every Monday and Thursday, $1 \mathrm{ml} / \mathrm{kg}$ each time. After 12 weeks, the LC model was verified according to liver biochemical indices and liver pathology. Then, the model mice were divided into 4 groups at random (based random order generator), and administered different treatments via the tail vein: (1) the HF-MSC group was injected with $1 \times 10^{6}$ HF-MSCs $(n=6)$; (2) the LV-HF-MSC group was injected with $1 \times 10^{6}$ 
LV-HF-MSCS $(n=6)$; (3) the ECM1-HF-MSC group was injected with $1 \times 10^{6}$ ECM1-HF-MSCs $(n=6)$; and (4) the model group was injected with an equivalent amount of saline $(n=6)$. Healthy mice $(n=6)$ were intraperitoneally injected with the same volume of saline. Four weeks after cell therapy, the liver tissue and serum of the sacrificed mice were collected for the subsequent analysis. Immunofluorescence was used to estimate the homing and differentiation of the transplanted cells in host liver tissues. Serum analysis was used to evaluate the recovery of liver function. Histological and molecular biological analyses were used to evaluate pathological recovery and the changes in pathway-related proteins.

\section{Activation of hepatic stellate cells and transwell assays}

The murine HSC cell line JS1 was purchased from Otwo Biotech (ShenZhen, China) and stimulated with TGF- $\beta 1$ [29] (Cat 100-21, $5 \mathrm{ng} / \mathrm{ml}$, Peprotech, USA) for $48 \mathrm{~h}$. Transwell chambers with an aperture of $0.4 \mu \mathrm{m}$ (Corning USA) placed on a 6-well plate were used to coculture HF-MSCs with activated JS1 cells. The cell concentration of the two kinds of cells was $2.5 \times 10^{5}$ cells $/ \mathrm{ml}$ after resuspension. Next, $1.5 \mathrm{ml}$ of an HF-MSC suspension was added to the upper chamber, and $2.6 \mathrm{ml}$ of a JS1 cell suspension was added to the lower chamber. After coculture with HF-MSCs for $48 \mathrm{~h}$, JS1 cells were collected for protein analysis.

\section{Western blot analysis}

Proteins that had been extracted from cells and liver tissue on ice with lysis solution were centrifuged. The supernatant was collected for concentration measurements and boiled for preservation or for subsequent experiments. The same amount of protein from each group was added to an SDS-PAGE gel and subjected to electrophoresis. The protein in the SDS-PAGE gel was transferred to a polyvinylidene fluoride (PVDF) membrane (Millipore, USA). Then, the membrane was blocked with $5 \%$ skimmed milk for $1.5 \mathrm{~h}$ at room temperature and incubated with primary antibodies (anti-ECM1 antibody, 11521-1-AP, 1:1000, Proteintech; anti-TGF- $\beta 1, \# 3711,1: 1500$, Cell Signaling Technology; anti- $\alpha-S M A$, sc-53142, 1:2000, Santa Cruz Biotechnology; anti-pSmad2/3, AF3367, 1:1000, Affinity; $\beta$-actin, ab8226, 1:1000, Abcam) at $4^{\circ} \mathrm{C}$ overnight. Thereafter, the membrane was further incubated with the corresponding secondary antibody (ab205718, 1:10000, Abcam) at $37^{\circ} \mathrm{C}$ for $50 \mathrm{~min}$. Enhanced chemiluminescence $(\mathrm{ECL})$ solution was used to visualize protein bands, and the relative protein level was calculated based on the housekeeping gene content with ImageJ.

\section{Immunofluorescence staining}

OCT-embedded liver sections held in an ultralow-temperature refrigerator were cut to a thickness of $6 \mu \mathrm{m}$ each and were left at room temperature for half an hour, rinsed with TBST to remove OTC on the surface and then fixed with cold acetone $\left(-20^{\circ} \mathrm{C}\right)$. An appropriate amount of goat blocking serum was used to block the liver sections for an hour. Primary antibodies (anti-CK18, ab181597, 1:200, Abcam; anti-ALB, ab207327, 1:500, Abcam; anti-AFP ab213328, 1:200, Abcam) were used to bind specific proteins on the sections at $4{ }^{\circ} \mathrm{C}$ overnight. The liver slices were then subsequently incubated with the secondary antibody (SA00013-4, 1:500, Proteintech) for an hour at $37^{\circ} \mathrm{C}$. The nuclei were stained with DAPI for $4 \mathrm{~min}$. After adding antifade mounting medium (Beyotime, China), the staining of the liver slices was observed under a fluorescence microscope (Olympus, Japan).

\section{Pathological analysis}

The tissue sections were successively transferred to different concentrations of xylene and ethanol for dewaxing and dyed in hematoxylin. Then the sections were rinsed with flowing water until they turned blue and dyed in eosin solution for 2 min. Thereafter, the stained sections were dehydrated with ethanol and treated with xylene. The transparent sections were dripped with neutral gum and sealed with a cover glass.

The paraffin sections were dewaxed and then successively washed with tap water and distilled water. The sections were subsequently stained with Weigert's iron hematoxylin staining solution for $5 \mathrm{~min}$. After differentiation with an acidic ethanol solution, Masson blue solution was used to restore the blue coloration. Then the sections were stained with Lichun red magenta staining solution for $5 \mathrm{~min}$, and the slices were sealed with neutral gum. Images of the slices were obtained with a BX51 microscope (Olympus, Japan).

\section{Analysis of liver biochemical indices in serum}

Blood samples of all groups were taken from the left ventricle for serological analysis when the mice were sacrificed. The obtained blood was placed in an EP tube and centrifuged. The supernatant was stored in an ultralow temperature freezer or used for subsequent experiments directly. The levels of alanine aminotransferase (ALT) (Cusabio, China), aspartate aminotransferase (AST) (Abcam), and alkaline phosphatase (ALP) (Cloud-Clone Corp, Wuhan, China) were measured according to the instructions to evaluate the liver function of each group.

\section{Statistical analyses}

At least three independent replicates were performed for each experiment. All the data are presented as the means $\pm S D$, and charts were generated using GraphPad Prism 8.0 (GraphPad Prism Software, San Diego, CA, USA). Data from more than two groups were analyzed using one-way analysis of variance (ANOVA), followed by Tukey's test, and two-tailed Student's $t$ test was used for comparisons between two groups. $F$ test was used to determine whether two population variances are equal. Non-parametric test was used for data that does not satisfy the normal distribution. $P<0.05$ was considered statistically significant.

\section{RESULTS}

\section{DEGs identification}

After normalizing the profile GSE103580 dataset, a total of 377 DEGs were obtained, $178(47.21 \%)$ of which were downregulated in LC, while 199 (52.79\%) were upregulated with a cutoff criterion of $P<0.05$ and a fold change $\geq 1.5$. Volcano plots and heat maps were used to present the screened DEGs (Fig. 2A, B).

\section{Identification of ECM1 as a target gene for HF-MSC transfection}

GO and KEGG analyses were used to enrich the biological processes and functions of DEGs. The DEGs were primarily enriched in pathways such as ECM-receptor interaction, focal adhesion, and chemical carcinogenesis (Fig. 2C, D). From what GO analysis represented (Fig. 2E, F), we observed that the DEGs were mainly related to the upregulation of extracellular structure organization and ECM organization. Considering that ECM plays a crucial role in LC, we took this as the starting point to identify foreign genes and aim to reduce ECM deposition.

Next, for the visualization of all DEG-encoded proteins, the online tools STRING and Cytoscape software were used to perform PPI network analysis of the DEGs. A total of 280 nodes and 847 edges in the aggregate were obtained in Cytoscape. In this network, we found the ECM1 gene, which is abundantly expressed in the ECM, was one of the DEGs downregulated in LC (Fig. 2G, H). Afterwards, we verified that ECM1 expression was decreased in LC mice relative to the healthy mice in vivo (Fig. 2I). Study also have shown that ECM1 can inhibit HSC activation [28]. Based on the above evidence, we choose ECM1 as the target gene for transfecting HF-MSCs.

\section{Identification of HF-MSCs and target gene expression in ECM1-HF-MSCs}

Primary HF-MSCs migrated across the culture plate and aggregated around the hair follicle bulge on the fourth day (Fig. 3A, B). As the cells proliferated, the third-generation HFMSCs fused into a monolayer and grew in a spindle shape (Fig. 3C). Oil red $\mathrm{O}$ staining and alizarin red staining confirmed that the HF-MSCs had the potential to differentiate into adipocytes and osteocytes (Fig. 3D, E). FACS analysis showed that the cell surface CD90 and CD29 (antigen phenotype of MSCs) expression was highest, while $<2 \%$ of HF-MSCs expressed CD43 (hematopoietic stem cell marker) and CD31 (endothelial cell surface marker) (Fig. $3 \mathrm{~F}-\mathrm{J}$ ). The above results indicated that most of the cells that we extracted were HFMSCs, with high purity. The preliminary results showed that the transfection efficiency of HF-MSCs was highest when the MOI was 40 (Fig. 3K). HF-MSCs that were GFP labeled and DAPI stained after infection at an optimal MOI of 40 are shown in Fig. 3L. A schematic diagram of the constructed lentiviral 
A

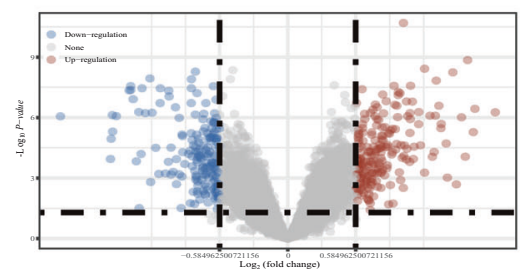

D

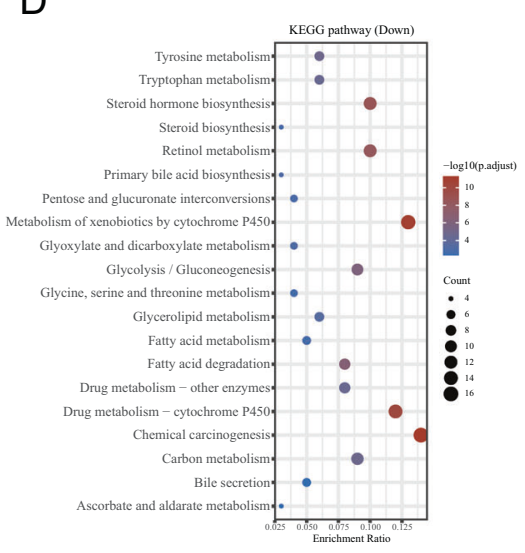

G

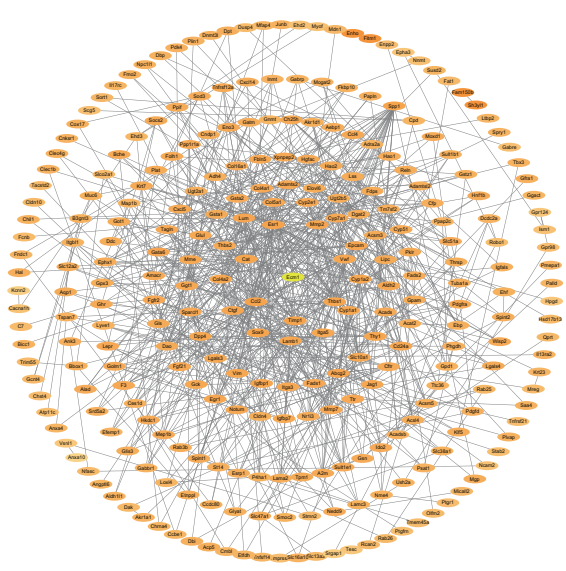

$E$

$\mathrm{H}$
B

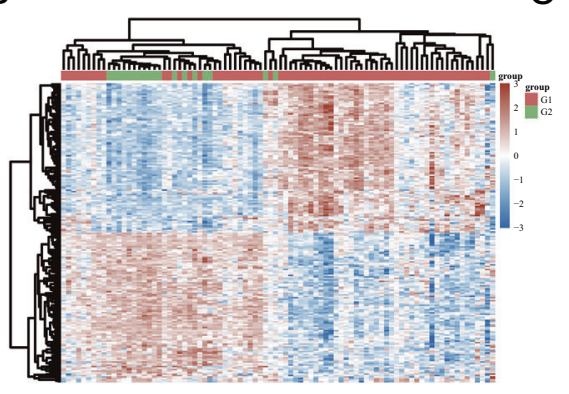

C
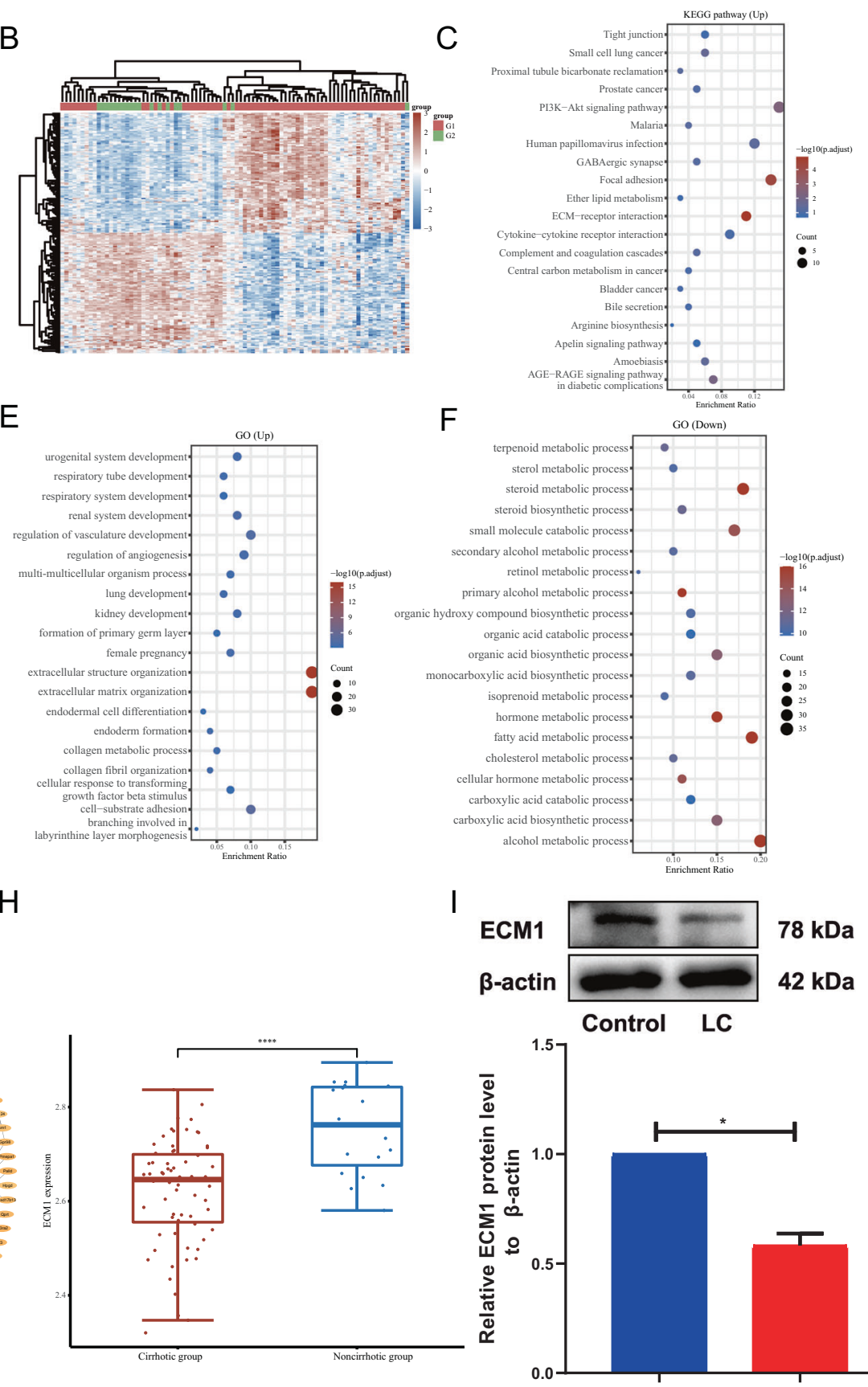

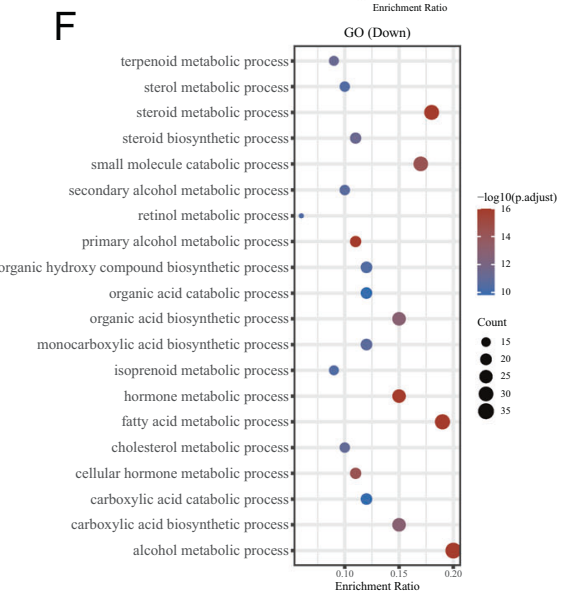

I

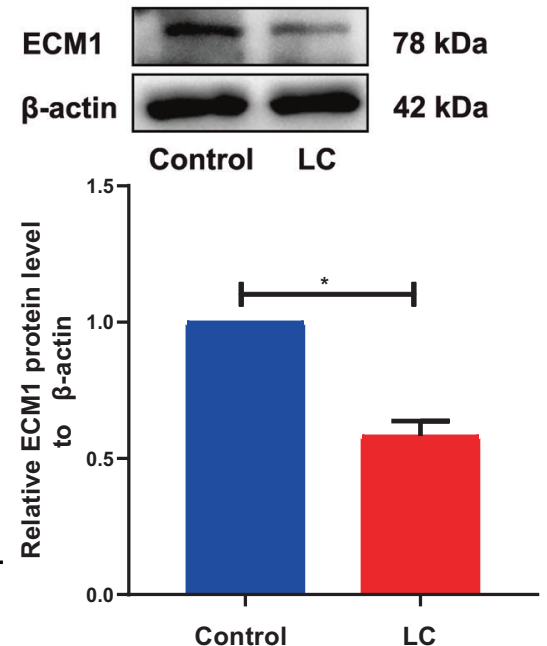

Fig. 2 DEG analysis and identification of ECM1 as the target gene. A Volcano plot of DEGs in GSE103580. B Heat map of DEGs in GSE103580. G1 represents the cirrhotic group; G2 represents the noncirrhotic group. Red indicates upregulation; green represents downregulation. KEGG pathway analysis of upregulated DEGs (C) and downregulated DEGs (D). GO enrichment of upregulated DEGs (E) and downregulated DEGs (F). G Protein-protein interaction networks among the DEGs. H Expression of ECM1 in DEGs. I Western blotting analysis and semiquantitative analysis of ECM1 expression in the control group and the model group. Data are shown as the means \pm SDs $\left({ }^{*} p<0.05,{ }^{* *} p<0.01,{ }^{* * *} p<0.001\right)$.

vector containing GFP, the target gene ECM1, and the puromycin resistance gene is shown in Fig. 3M. ECM1 was shown to be overexpressed in ECM1-HF-MSCs relative to HFMSCs and LV-HF-MSCs in qualitative and semiquantitative analyses (Fig. 3N, O).

\section{Verification of the LC model in mice}

In the 12th week after the injection of $\mathrm{CCl}_{4}$, liver pathology and liver biochemical indices were used to evaluate the model establishment. The staining of the pathological section shows that there was no obvious abnormality detected in the heathy mice, while in the LC group, there was a degeneration and necrosis of hepatocytes, with a large area exhibiting pseudolobule structure. Additionally, large amounts of fibrous tissues proliferated and were connected with each other, and these tissues separated and wrapped the liver tissue to form pseudolobules (Fig. 4A, B). In addition, after induction by $\mathrm{CCl}_{4}, \mathrm{AST}, \mathrm{ALT}$ and ALP levels were several times higher than those in healthy mice (Fig. 4C-E). These results indicated that a 12-week modeling cycle can lead to LC in mice. 

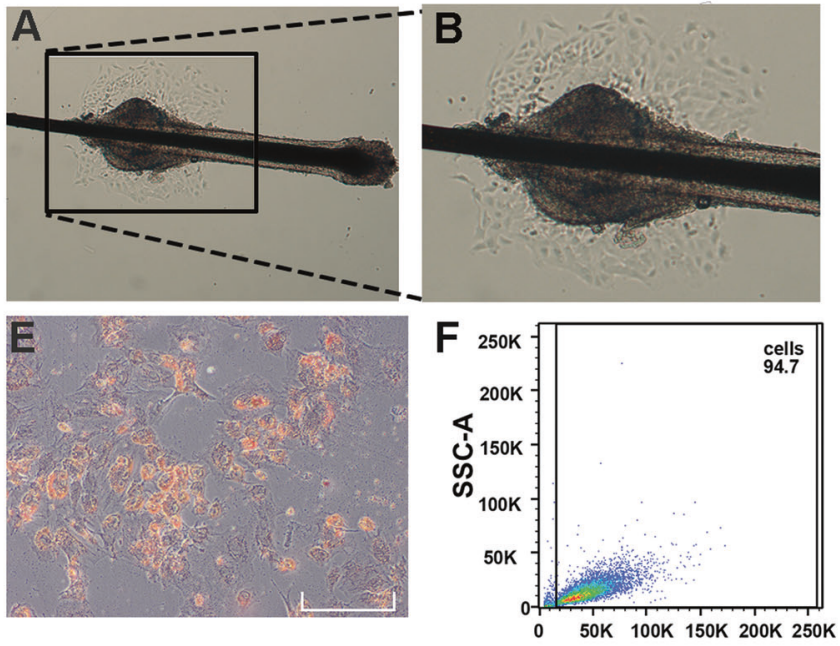

FSC-A

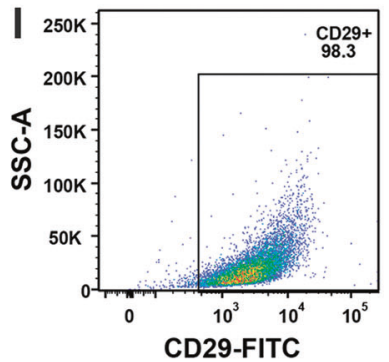

M
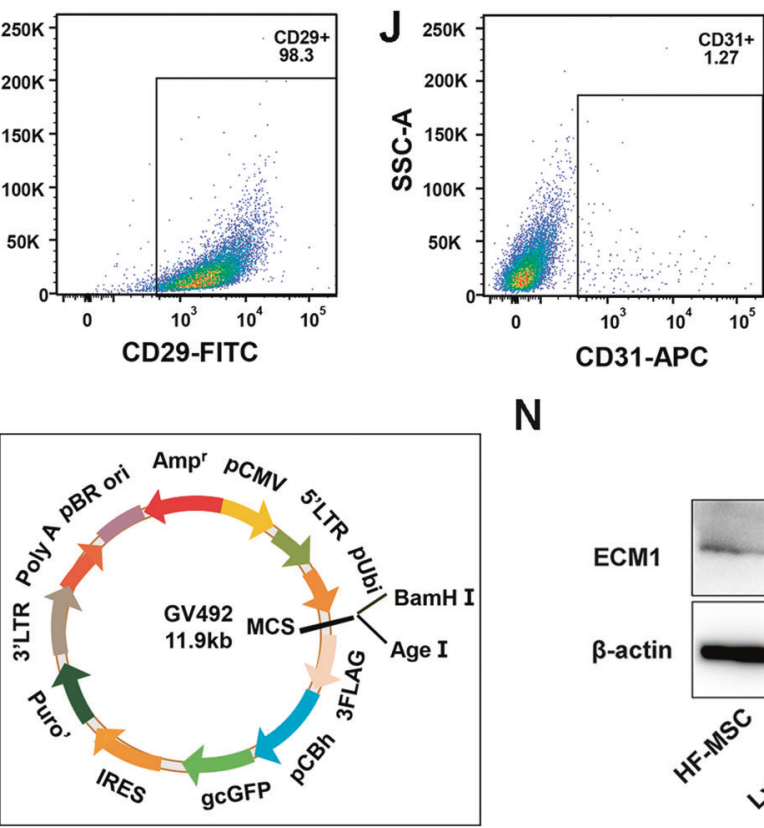

$\mathbf{N}$
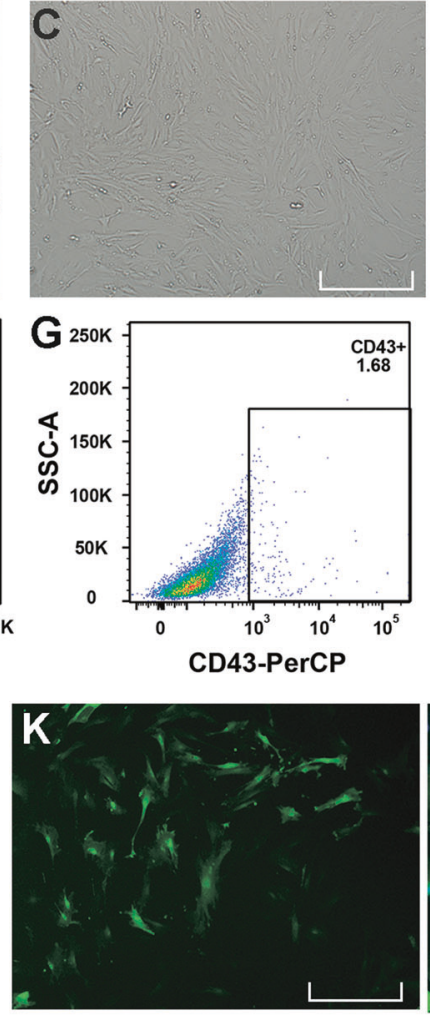
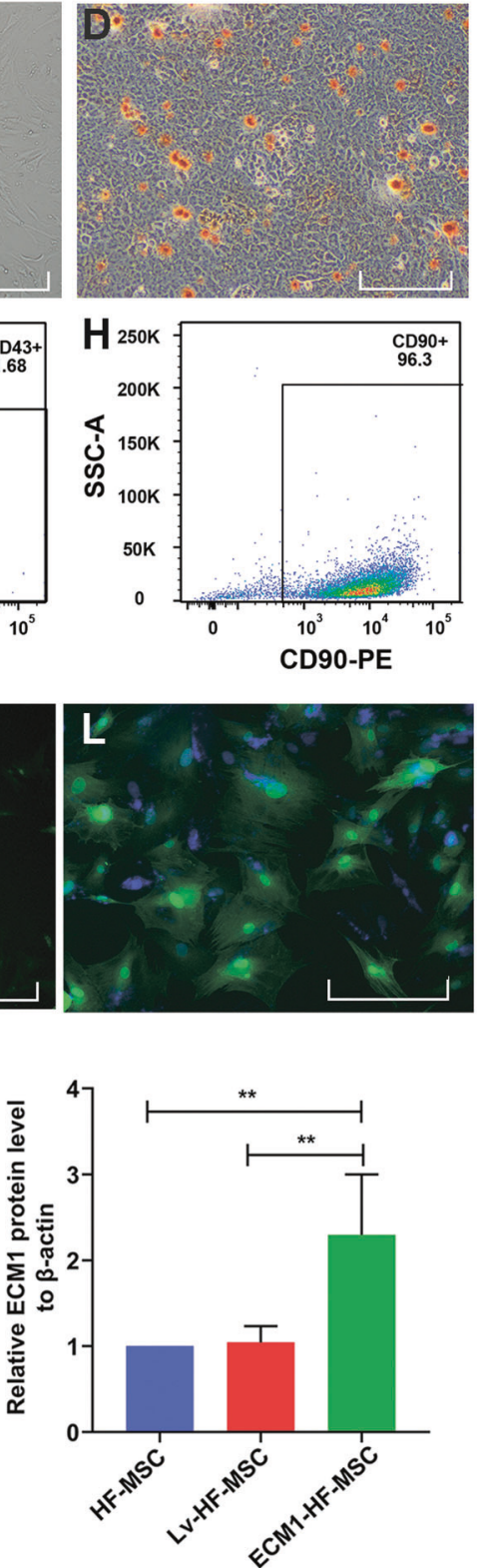

Fig. 3 Identification and transfection of HF-MSCs. Primary (A, B) and P3 (C) HF-MSCs. D, E Adipogenic and osteogenic differentiation of HFMSCs. F-J Identification of specific antigenic markers in HF-MSCs by flow cytometry. $\mathbf{K}$ The transfected HF-MSCs show green fluorescence under a 4X microscope. L HF-MSCs expressing GFP were costained for nuclear detection and observed under a 10X microscope. $\mathbf{M}$ The constructed lentiviral vector containing ECM1 and GFP. N, $\mathbf{O}$ Immunoblotting analysis and semiquantitative analysis of ECM1 in the HFMSC group, the LV-HF-MSC group, and the ECM1-HF-MSC group. Data are shown as the means \pm SDs $\left({ }^{*} p<0.05,{ }^{* *} p<0.01,{ }^{* * *} p<0.001\right)$. Scale bar $=50 \mu \mathrm{m}$.

\section{ECM1-HF-MSCs inhibit the activation of HSCs by blocking the TGF- $\beta$ /Smad pathway in vitro}

The unactivated JS1 cells were polygonal under an inverted phase-contrast microscope (Fig. 5A). After $48 \mathrm{~h}$ of TGF$\beta 1$ stimulation [29], the activated JS1 cells showed a long fusiform shape (Fig. 5B) and were used for subsequent experiments. To detect the activation of JS1 cells and changes in pathway proteins, JS1 cells were collected after coculture with HF-MSCs, LV-HF-MSCs, and ECM1-HF-MSCs for $48 \mathrm{~h}$. The a-SMA expression was suppressed in cell therapy groups in contrast to the JS1 group, while the expression in the ECM1-HF-MSC group was the lowest. Significantly decreased TGF- $\beta 1$ and $p-S m a d 2 / 3$ levels were observed in the treatment groups, and the expression level was the lowest in the ECM1-HF-MSC group (Fig. 5C-G). These results indicated that ECM1-HF-MSCs have a greater ability than naïve HF-MSCs to inhibit the activation of JS1 cells through the TGF- $\beta /$ Smad pathway.

\section{Grafted HF-MSCs exist in the liver and express hepatocyte-} specific surface markers

In the 4th week after ECM1-HF-MSC transplantation, we detected the distribution of GFP-stained HF-MSCs in the liver, intestine, kidney, lung and spleen by immunofluorescence. As shown in Fig. 6A, E, I, HF-MSCs were detected in the injured liver but were barely observable in the intestine, kidney, lung, and spleen (Fig. 6Q-T). In addition, we found that the transplanted GFP-labeled ECM1-HF-MSCs 

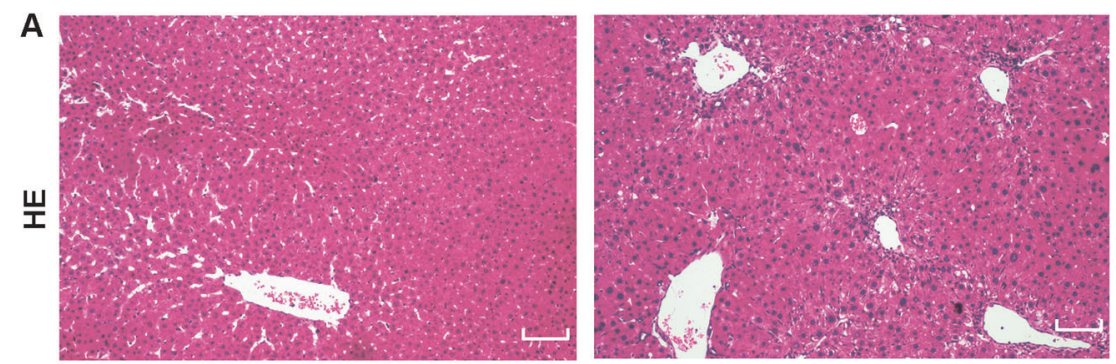

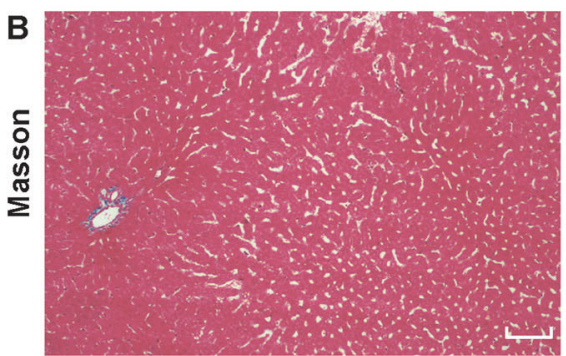

Control

C

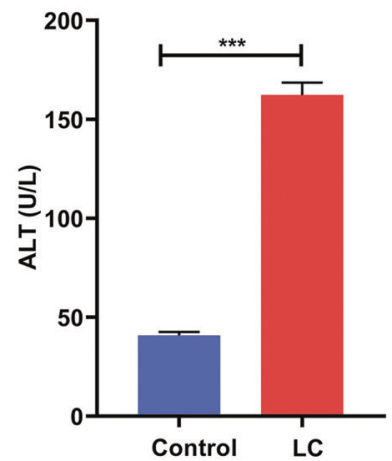

D

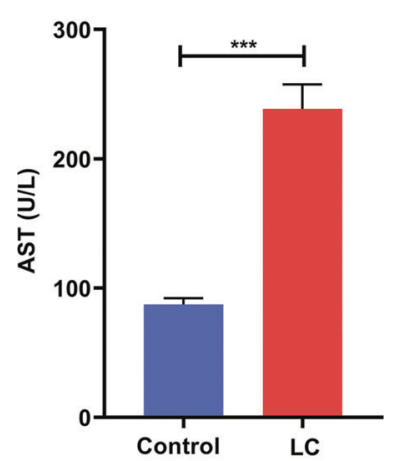

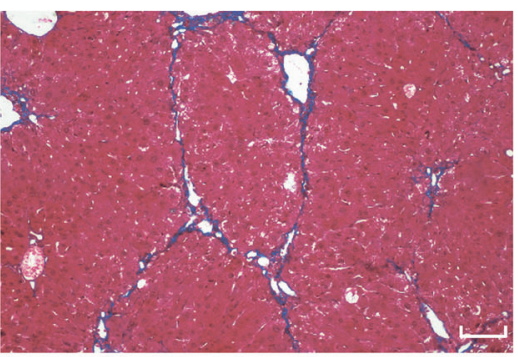

LC

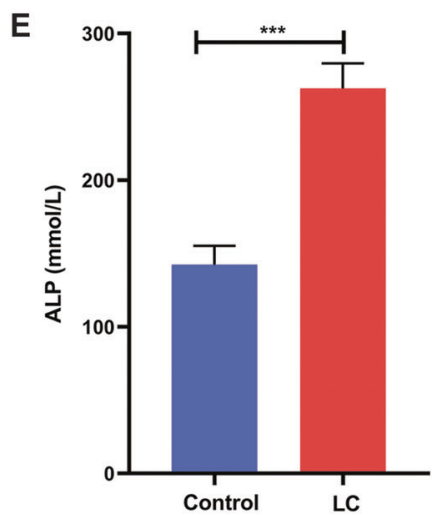

Fig. 4 Verification of the LC model. A, B HE staining and Masson staining in the control group and the LC group. C, D, E Comparison of the liver serological indices of ALT, AST, and ALP in the control group and the LC group. Scale bar $=50 \mu \mathrm{m}$.

were costained with $C K 18, A L B$, and AFP, which are hepatocytespecific surface markers (Fig. 6B, F, J). It indicated that ECM1-HF-MSCs differentiated into hepatocyte-like cells (HLCs) (Fig. 6D, H, L). Pearson's correlation, overlap coefficient and scatter plot, which reflect the degree of the colocalization of GFP and hepatocytespecific surface markers, are shown in Fig. 6M-P.

\section{Transplantation of ECM1-HF-MSCs show a better effect on promoting injured liver repair and improving liver function} $\mathrm{HE}$ and Masson staining were used to evaluate liver pathological changes after cell treatments. It showed that the lobules and portal areas were normal in the control group, while large areas of proliferative fibrous tissue divided and wrapped the hepatocyte regeneration nodules into pseudolobules of different sizes in the model group. However, the typical pathological features of cirrhosis were significantly improved after cell therapy, of which the most significant improvement was achieved by the administration of ECM1-HF-MSCs (Fig. 7A, B). In addition, the area of collagen fibers was reduced by cell treatment compared with that in the LC mice, while that in the ECM1-HF-MSC group was smallest (Fig. 7C). The Ishak score was used to detect damage to liver tissue in each group. The model group showed the most serious damage, and the ECM1-HF-MSC group showed the most obvious remission (Fig. 7D).

A serological assay was used to detect liver function in each group. The high levels of AST, ALT and ALP in the LC mice were reversed by cell treatment and showed different downward trends. It is worth mentioning that the serological indices in the ECM1-HF-MSC group tended to be normal (Fig. 7E-G). The above results showed that ECM1-HF-MSCs can improve $\mathrm{CCl}_{4}$-induced LC more effectively than other treatments in terms of both pathology and liver function.

\section{ECM1-transfected HF-MSCs inhibit the activation of HSCs via the TGF- $\beta$ /Smad pathway in vivo}

We detected HSC activation at the protein level in vivo. The a-SMA level was remarkably inhibited by cell treatment in comparison with the model group, and the inhibition was most significant in the ECM1-HF-MSC group (Fig. 7H, I).

The remarkable tendency of high TGF- $\beta$ and $p-S m a d 2 / 3$ expression observed in the LC model was reversed by cell transplantation treatment. The TGF- $\beta$ and $p$-smad $2 / 3$ levels in liver tissue among the treatment groups showed marked differences, and the protein levels in the ECM1-HF MSC group presented a more downward trend than other treatment groups (Fig. $7 \mathrm{H}, \mathrm{J}, \mathrm{K}$ ). This finding indicated that the overexpression of ECM1 in HF-MSCs resulted in greater inhibition of HSC activation via the TGF- $\beta / S m a d$ pathway than naïve HF-MSCs.

\section{DISCUSSION}

In this study, we used bioinformatic tools to screen the HSCrelated target gene ECM1 from the DEGs identified in LC and then 
A

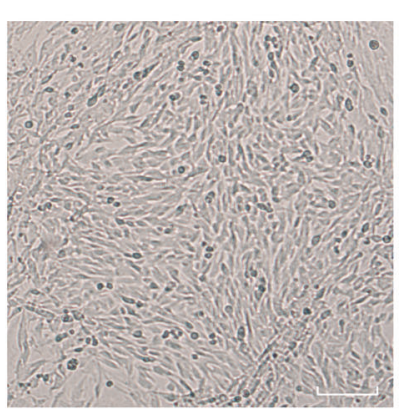

Quiescent HSC
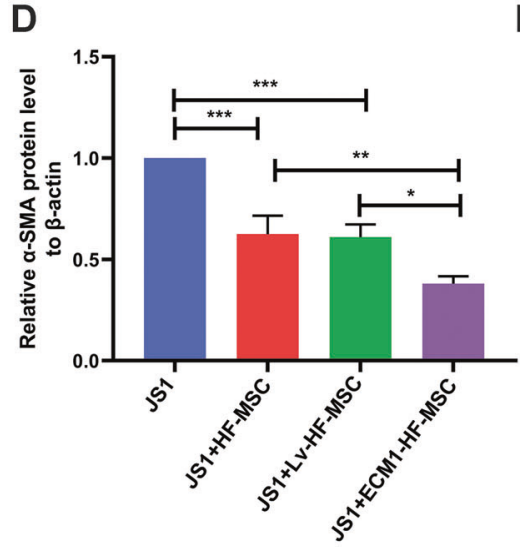

B

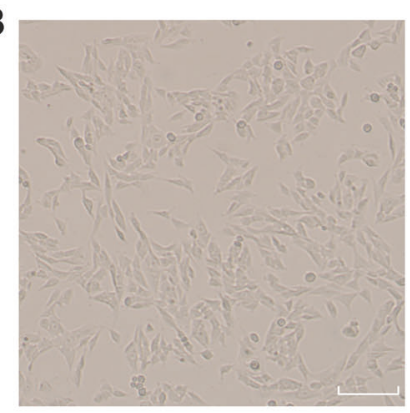

Activated HSC

E

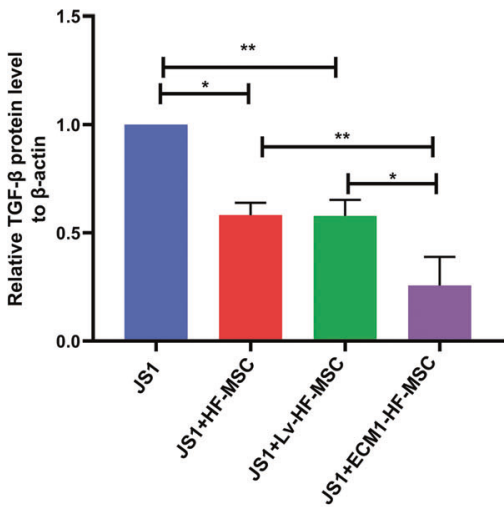

C

F
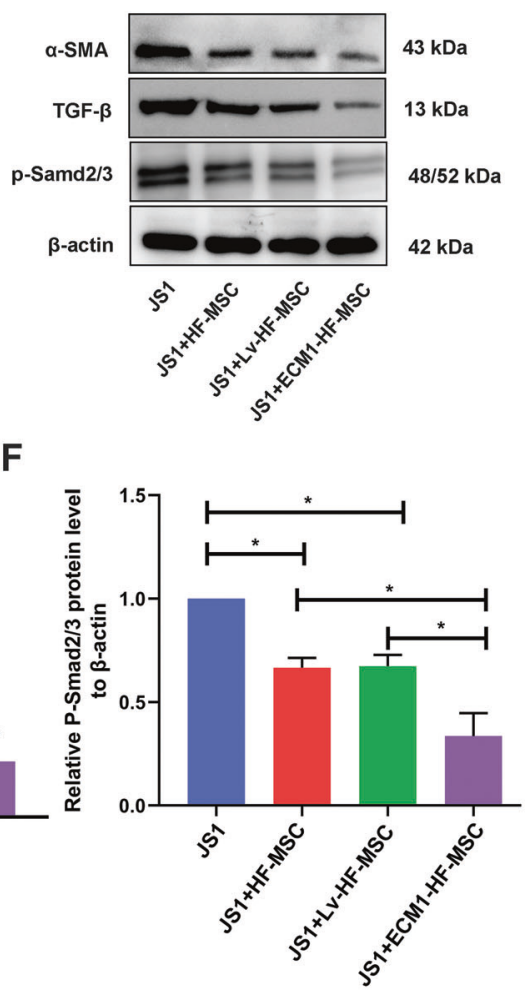

Fig. 5 ECM1-HF-MSCs inhibit the TGF- $\beta$ /Smad signaling pathway and activation of HSCs in vitro. A The quiescent murine hepatic stellate cell line JS1. B Activated JS1 cells. Scale bar $=50 \mu \mathrm{m}$. C Western blotting analysis of a-SMA, TGF- $\beta$, and $\mathrm{p}-\mathrm{Smad} 2 / 3$ in all groups. D-F Semiquantitative analysis of the pathway proteins and a-SMA. Data are shown as the means \pm SDs $\left({ }^{*} p<0.05,{ }^{* *} p<0.01,{ }^{* * *} p<0.001\right)$.

transfected it into HF-MSCs. We found that ECM1-transfected HFMSCs represented a more effective treatment for LC in terms of restoring pathology and liver function than naïve HF-MSCs, which may be mediated by multiple mechanisms.

The LC model was established with $\mathrm{CCl}_{4}$, and its characteristics were very similar to the pathological characteristics of human drug-induced LC [30]. The method of $\mathrm{CCl}_{4}$ application in our research was intraperitoneal injection, which results in a high modeling rate and survival rate, with good reproducibility [31]. Twelve weeks after the intraperitoneal injection of $\mathrm{CCl}_{4}$, the mouse liver pathological and serological analysis showed that typical pseudolobules formed, and the serum indices were significantly increased, which indicated that we successfully established the LC model in mice.

Cirrhosis often leads to death due to various complications, and an effective treatment is currently lacking. MSC transplantation has been shown to improve liver fibrosis, even in the advanced stages of LC [5, 32]. In addition, MSCs are a good vector that can be easily transfected with retroviruses and lentiviruses, with transfection rates of $50-80 \%$ [33], and these retroviruses and lentiviruses are commonly used for the overexpression of foreign transgenes [34]. We transfected ECM1 into HF-MSCs with lentivirus to overexpress ECM1. After cell transplantation, we found that liver function was improved and that the content of collagen fibers was decreased by cell therapy, especially by ECM1-HF-MSC treatment.

Multiple mechanisms may be involved in the therapeutic effect of ECM1-HF-MSCs on LC. This effect requires a sufficient number of MSCs to home to the injured tissue and exert anti-inflammatory or differentiation effects to repair injured tissues [35]. SDF-1 is a widely studied chemokine involved in homing. When tissue damage occurs, SDF-1 expression in damaged cells increases, and
MSCs are then recruited via the chemoattraction imposed by an SDF- 1 concentration gradient and are retained at the injured site, which exhibits a high SDF concentration [36]. It shows that BMMSCs can home to damaged tissues and survive in these tissues for up to 13 months [37, 38]. Similarly, we found that GFP-labeled ECM1-HF-MSCs existed in the damaged liver tissue 4 weeks after cell transplantation. However, we only detected the homing of ECM1-HF-MSCs in the 4th week after cell transplantation. The homing efficiency of ECM1-HF-MSCs at different times can be further investigated in subsequent studies, and more effective methods for enhancing the chemotaxis of MSCs to the damaged liver can be explored.

Previous studies suggest that MSCs can differentiate into HLCs regardless of their origin [39-41], which can restore hepatocyte vitality when liver injury occurs. In our research, ECM1-HF-MSCs labeled with GFP in liver tissue expressed the hepatocyte-specific markers CK18, ALB and AFP, which indicated that ECM1transfected HF-MSCs show a tendency to differentiate into HLCs. It requires strict conditions and the participation of a variety of cytokines for MSCs to different into hepatocytes. In addition, the Wnt pathway and epigenetic modifications also contributed to this process $[42,43]$. However, the therapeutic effect of MSCs on LC is affected by the differentiation efficiency of MSCs [4]. More strategies to improve the differentiation efficiency of HF-MSCs need to be studied.

Next, we studied the effect of ECM1-HF-MSCs on the fate of HSCs. In the case of liver injury, HSCs with a dormant phenotype become myofibroblasts, which are the main source of ECM components in pathological fibrous tissue [44]. Yu F et al. [45] proved that MSCs inhibit HSC activation after coculture with MSCs in vitro. Here, we also found that HF-MSCs could reverse the 

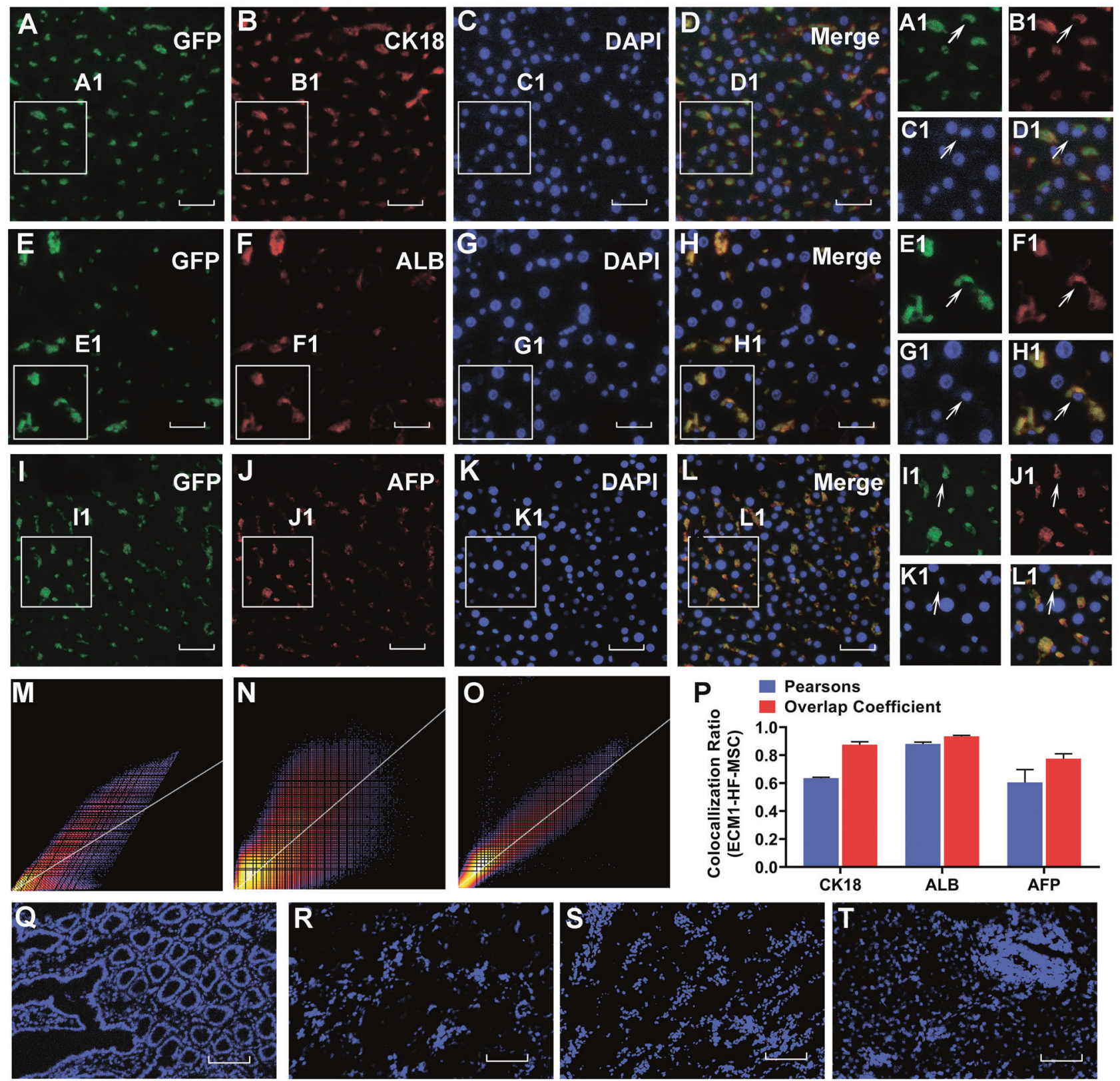

Fig. 6 ECM1-overexpressing HF-MSCs home to the injured liver site and differentiate into hepatocyte-like cells. Colocalization of GFPexpressing ECM1-HF-MSCs and the hepatic-specific surface markers CK18 A-D, ALB E-H, and AFP I-L. A1-L1 Magnification of regions of (A-L). M-O Scatter plots of GFP-expressing ECM1-HF-MSCs and CK18, ALB, and AFP levels. P Pearson's correlation and the overlap coefficient of liver sections costained for CK18, ALB, and AFP in the ECM1-HF-MSC group. Q-T GFP-labeled ECM1-HF-MSCs were rarely observed in the intestine, kidney, lung, or spleen. Scale bar (A-L), $50 \mu \mathrm{m}$; Scale bar (Q-T), $200 \mu \mathrm{m}$.

activation of HSCs, while ECM1-HF-MSCs exerted a stronger inhibitory effect on HSCs. The effect of MSCs on HSCs could be realized by direct contact between the two kinds of cells or by transmitting signals to HSCs through cytokines secreted by MSCs to change the fate of HSCs. On the other hand, MSCs can exert indirect effects on HSCs by acting on immune cells, the immune response was reduced due to the immunosuppressive properties of MSCs, thereby reducing the pro-fibrotic stimulation of immune cells to HSCs [46]. It is widely accepted that the MSCs anti-fibrotic effect is mainly due to the paracrine factors $[47,48]$, the specific mechanism of HF-MSCs on HSCs deserves further exploration.

Liver fibrosis and cirrhosis develop under the action of common signaling pathways, although the etiology of liver disease may be different. TGF- $\beta 1 /$ Smad pathway activation is essential in liver fibrosis $[49,50]$. Research proved that MSCs can alleviate LC by regulating the TGF- $\beta 1 / \mathrm{Smad}$ pathway [51], which is in line with our results. TGF- $\beta$ exists as a latent complex in the liver, and av integrin plays a role in interacting with latent TGF- $\beta$ to convert it into activated TGF- $\beta$, leading to the development of $L C[52,53]$. As shown in Fig. 8, ECM1 interacts with $a_{v}$ integrin, which disturbs TGF- $\beta$ activation to maintain liver homeostasis [54]. When ECM1 is reduced due to liver injury, TGF- $\beta$ is activated, and the activation of HSCs is initiated. The trend is reversed when exogenous ECM1 is administered [28]. In our study, ECM1-HF-MSCs continuously supplied exogenous ECM1, thereby stably inhibiting the activation of TGF- $\beta$ and HSCs. This explains why TGF- $\beta 1$ and $p-S m a d 2 / 3$ 


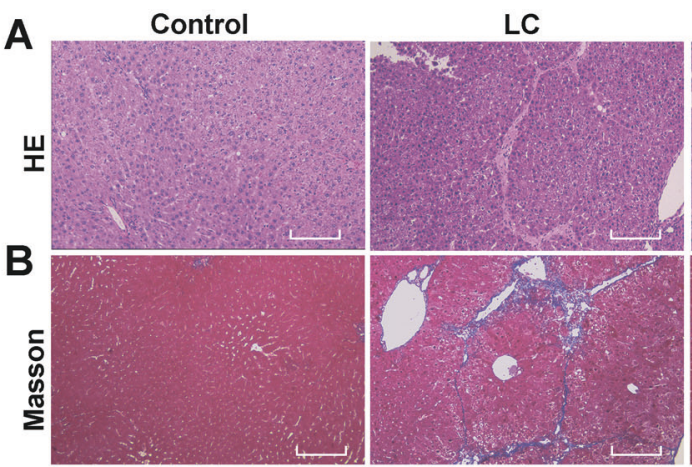

C

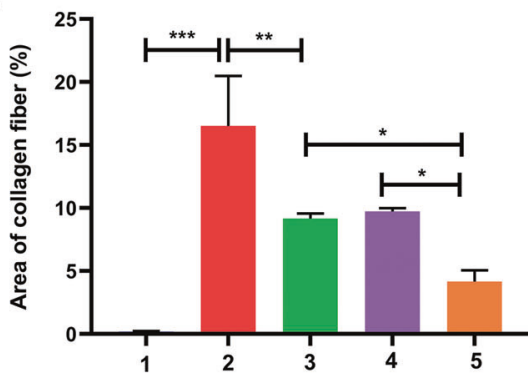

E

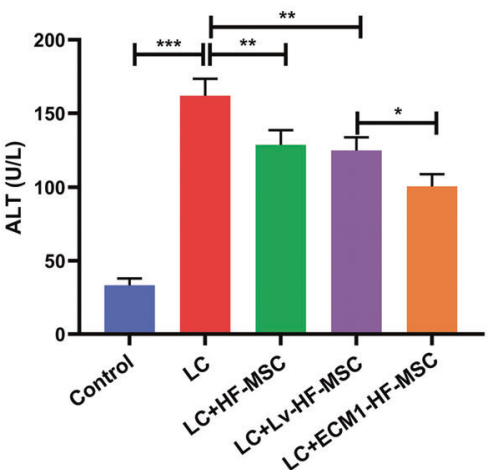

H

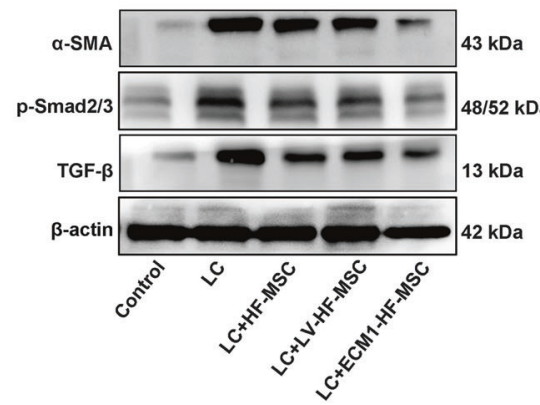

1: Control

2: LC

3: LC+HF-MSC

4: LC+Lv-HF-MSC

5: LC+ECM1-HF-MSC
LC+HF-MSC
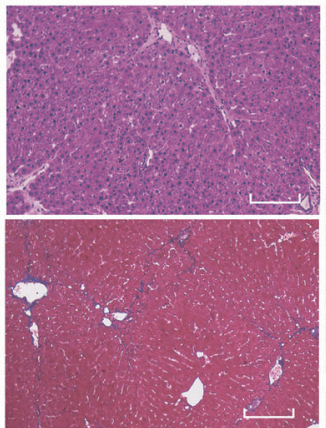

D

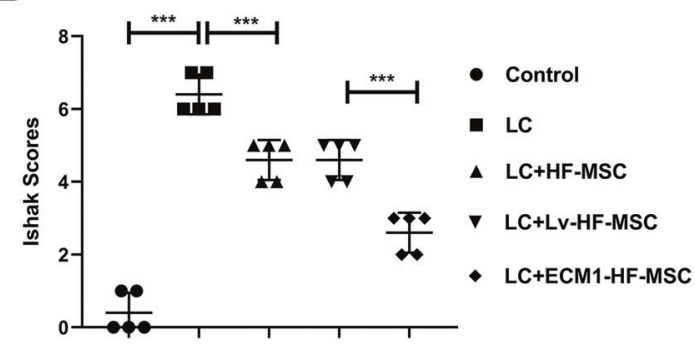

LC+Lv-HF-MSC
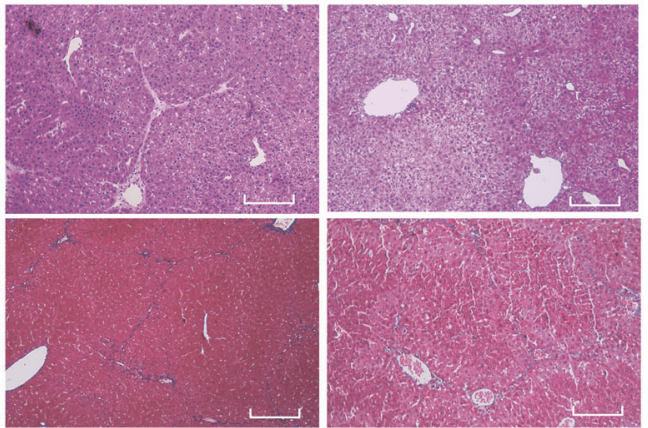

LC+ECM1-HF-MSC
$F$

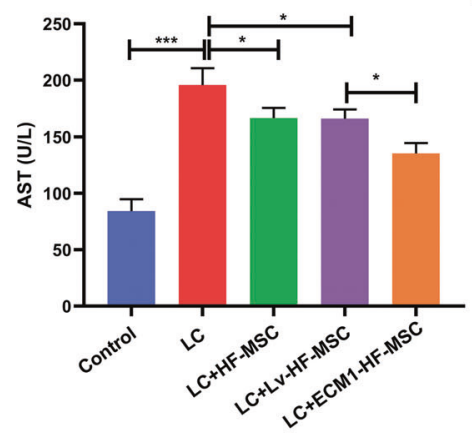

I

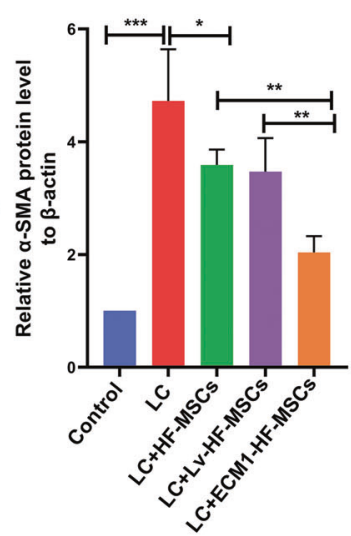

J
G

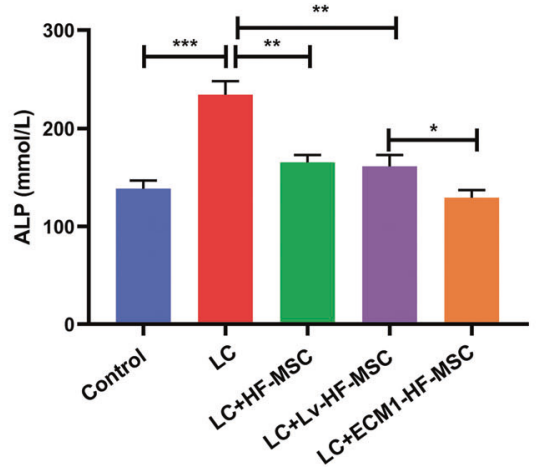

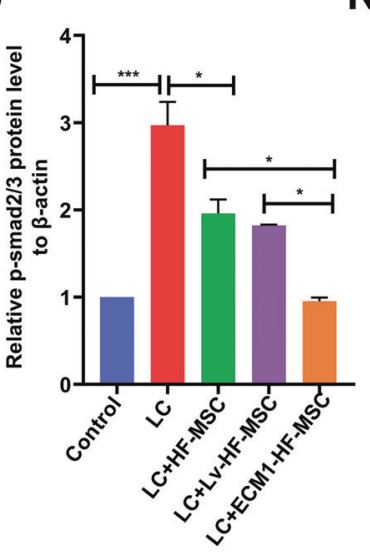

K

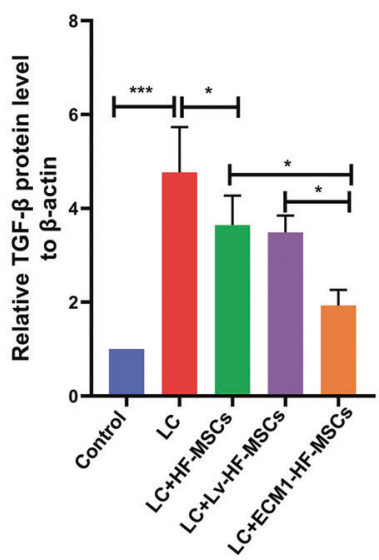

Fig. 7 ECM1-HF-MSCs show a better effect in LC treatment through inhibiting the activation of HSCs and the TGF- $\beta /$ Smad signaling pathway in vivo. A, B HE and Masson staining in each group. Scale bar $=100 \mu \mathrm{m}$. C Area of collagen fibers in each group. D Ishak scores show the degree of LC in all groups. E-G Changes in the serological indices of ALT, AST and ALP. H-K Western blotting and semiquantitative analysis of a-SMA, $\mathrm{p}-\mathrm{Smad} 2 / 3$, and TGF- $\beta$ in all groups. Data are shown as the means \pm SDs $\left({ }^{*} p<0.05,{ }^{* *} p<0.01,{ }^{* * *} p<0.001\right)$.

levels in the ECM1-HF-MSC group were the lowest among all cell treatment groups.

In summary, this study showed that ECM1 modified HF-MSCs have the potential to migrate to the injured liver and differentiate in to HLCs. ECM1-HF-MSCs showed a more significant therapeutic effect on LC than naïve HF-MSCs, which may be mediated most likely by the inhibition of HSC pathological activation via the TGF$\beta /$ Smad pathway. Our research may provide evidence for the combination of bioinformatic technology and genetic engineering to achieve precise treatment of LC. 


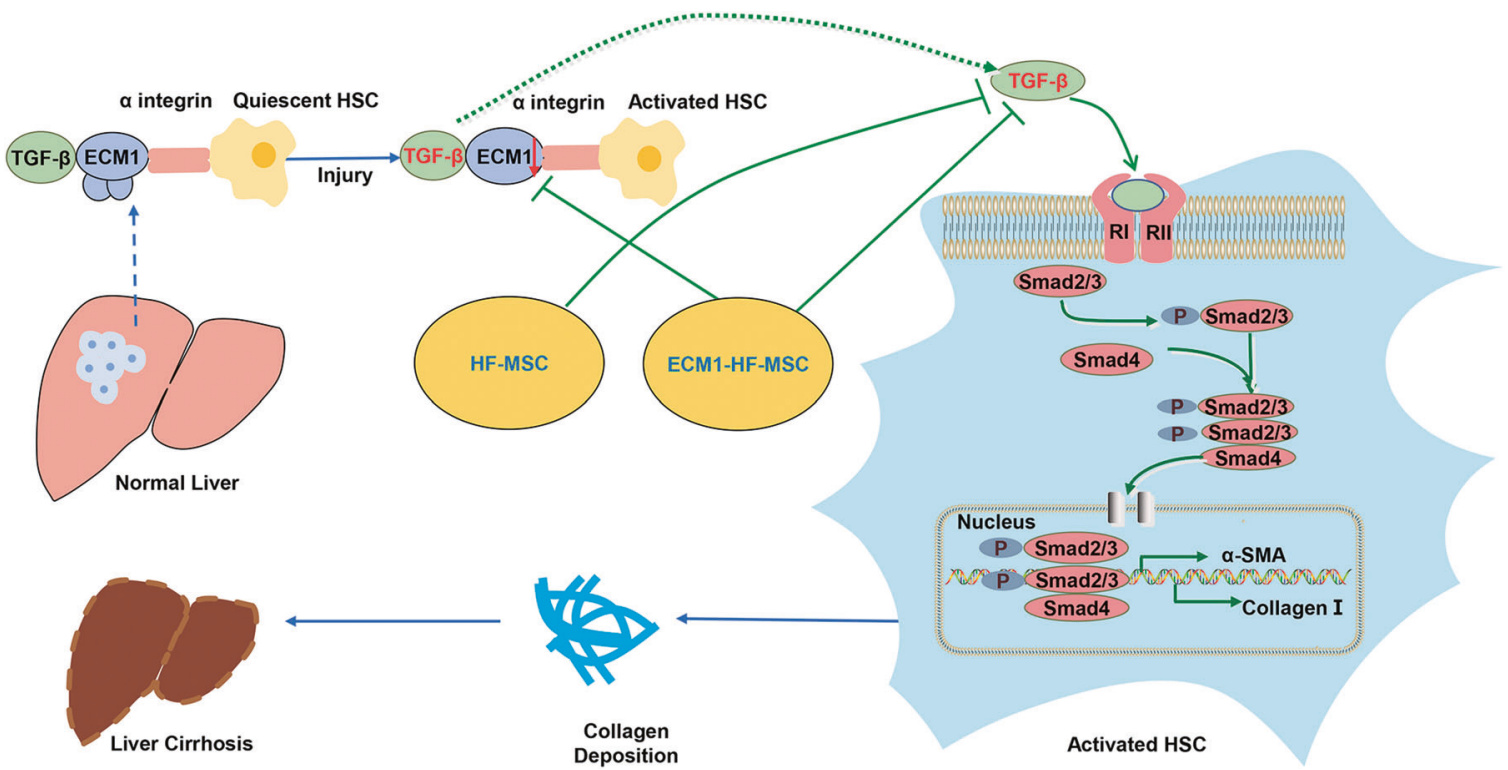

Fig. 8 Overview diagram describing the mechanism of the inhibitory effect of HF-MSCs on HSCs. In a healthy liver, ECM1 interacts with $\alpha \mathrm{V}$ integrin to maintain the latent state of TGF- $\beta$. After liver injury, ECM1 levels are decreased, leading to the activation of TGF- $\beta$ by $\alpha_{V}$ integrin, which in turn activates HSCs through the TGF- $\beta$ /Smad pathway. ECM1-HF-MSCs can inhibit the activation of TGF- $\beta$ through exogenous supplementation with ECM1, which in turn affects the activation of HSCs, thereby alleviating LC.

\section{DATA AVAILABILITY}

The data that support the findings of this study are available on request from the corresponding author.

\section{REFERENCES}

1. Guo Y, Chen B, Chen LJ, Zhang CF, Xiang C. Current status and future prospects of mesenchymal stem cell therapy for liver fibrosis. J Zhejiang Univ Sci B. 2016;17:831-41.

2. Goldberg D, French B, Trotter J, Shetty K, Schiano T, Reddy KR, et al. Underreporting of liver transplant waitlist removals due to death or clinical deterioration: results at four major centers. Transplantation.2013;96:211-6.

3. Terai S, Takami T, Yamamoto N, Fujisawa K, Ishikawa T, Urata Y, et al. Status and prospects of liver cirrhosis treatment by using bone marrow-derived cells and mesenchymal cells. Tissue Eng Part B Rev. 2014;20:206-10.

4. Dai LJ, Li HY, Guan LX, Ritchie G, Zhou JX. The therapeutic potential of bone marrow-derived mesenchymal stem cells on hepatic cirrhosis. Stem Cell Res. 2009;2:16-25.

5. Lee UE, Friedman SL. Mechanisms of hepatic fibrogenesis. Best Pr Res Clin Gastroenterol. 2011;25:195-206.

6. Friedman SL. Evolving challenges in hepatic fibrosis. Nat Rev Gastroenterol Hepatol. 2010;7:425-36.

7. Bataller R, Brenner DA. Liver fibrosis. J Clin Invest. 2005;115:209-18.

8. Kubo K, Ohnishi S, Hosono H, Fukai M, Kameya A, Higashi R, et al. Human amnionderived mesenchymal stem cell transplantation ameliorates liver fibrosis in rats. Transpl Direct. 2015;1:e16.

9. Cotsarelis G. Epithelial stem cells: a folliculocentric view. J Invest Dermatol. 2006;126:1459-68.

10. Liu JY, Peng HF, Andreadis ST. Contractile smooth muscle cells derived from hairfollicle stem cells. Cardiovasc Res. 2008;79:24-33.

11. Bajpai VK, Mistriotis $P$, Andreadis ST. Clonal multipotency and effect of long-term in vitro expansion on differentiation potential of human hair follicle derived mesenchymal stem cells. Stem Cell Res. 2012;8:74-84.

12. Shi X, Lv S, He X, Liu X, Sun M, Li M, et al. Differentiation of hepatocytes from induced pluripotent stem cells derived from human hair follicle mesenchymal stem cells. Cell Tissue Res. 2016;366:89-99.

13. Roh C, Tao Q, Lyle S. Dermal papilla-induced hair differentiation of adult epithelial stem cells from human skin. Physiol Genomics. 2004;19:207-17.

14. Sieber-Blum M, Grim M, Hu YF, Szeder V. Pluripotent neural crest stem cells in the adult hair follicle. Dev Dyn. 2004;231:258-69.

15. Taura D, Noguchi M, Sone M, Hosoda K, Mori E, Okada Y, et al. Adipogenic differentiation of human induced pluripotent stem cells: comparison with that of human embryonic stem cells. FEBS Lett. 2009;583:1029-33.
16. di Bonzo LV, Ferrero I, Cravanzola C, Mareschi K, Rustichell D, Novo E, et al. Human mesenchymal stem cells as a two-edged sword in hepatic regenerative medicine: engraftment and hepatocyte differentiation versus profibrogenic potential. Gut.2008;57:223-31.

17. Forbes SJ, Russo FP, Rey V, Burra P, Rugge $M$, Wright NA, et al. A significant proportion of myofibroblasts are of bone marrow origin in human liver fibrosis. Gastroenterology.2004;126:955-63.

18. Ye Z, Lu W, Liang L, Tang M, Wang $Y$, Li Z, et al. Mesenchymal stem cells overexpressing hepatocyte nuclear factor- 4 alpha alleviate liver injury by modulating anti-inflammatory functions in mice. Stem Cell Res Ther. 2019;10:149.

19. Kim JY, Choi JH, Jun JH, Park S, Jung J, Bae SH, et al. Enhanced PRL-1 expression in placenta-derived mesenchymal stem cells accelerates hepatic function via mitochondrial dynamics in a cirrhotic rat model. Stem Cell Res Ther. 2020;11:512.

20. Moon SH, Lee CM, Park SH, Jin, Nam M. Effects of hepatocyte growth factor genetransfected mesenchymal stem cells on dimethylnitrosamine-induced liver fibrosis in rats. Growth Factors. 2019;37:105-19.

21. Qu Y, Zhang Q, Cai X, Li F, Ma Z, Xu M, et al. Exosomes derived from miR-181-5pmodified adipose-derived mesenchymal stem cells prevent liver fibrosis via autophagy activation. J Cell Mol Med. 2017;21:2491-502.

22. Kim MD, Kim SS, Cha HY, Jang SH, Chang DY, Kim W, et al. Therapeutic effect of hepatocyte growth factor-secreting mesenchymal stem cells in a rat model of liver fibrosis. Exp Mol Med. 2014;46:e110.

23. Mathieu E, Meheus L, Raymackers J, Merregaert J. Characterization of the osteogenic stromal cell line MN7: identification of secreted MN7 proteins using two-dimensional polyacrylamide gel electrophoresis, western blotting, and microsequencing. J Bone Min Res. 1994;9:903-13.

24. Deckers MM, Smits $P$, Karperien $M, N i$ J, Tylzanowski $P$, Feng $P$, et al. Recombinant human extracellular matrix protein 1 inhibits alkaline phosphatase activity and mineralization of mouse embryonic metatarsals in vitro. Bone.2001;28:14-20.

25. Han Z, Ni J, Smits P, Underhill CB, Xie B, Chen Y, et al. Extracellular matrix protein 1 (ECM1) has angiogenic properties and is expressed by breast tumor cells. FASEB J. 2001;15:988-94.

26. Wang $\mathrm{L}$, Yu J, Ni J, Xu XM, Wang J, Ning $\mathrm{H}$, et al. Extracellular matrix protein 1 (ECM1) is over-expressed in malignant epithelial tumors. Cancer Lett. 2003;200:57-67.

27. Smits $P$, Poumay $Y$, Karperien M, Tylzanowski $P$, Wauters J, Huylebroeck D, et al. Differentiation-dependent alternative splicing and expression of the extracellular matrix protein 1 gene in human keratinocytes. J Invest Dermatol. 2000;114:718-24.

28. Fan W, Liu T, Chen W, Hammad S, Longerich T, Hausser I, et al. ECM1 prevents activation of transforming growth factor beta, hepatic stellate cells, and fibrogenesis in mice. Gastroenterology.2019;157:1352-67.

29. Ma L, Zeng Y, Wei J, Yang D, Ding G, Liu J, et al. Knockdown of LOXL1 inhibits TGFbeta1-induced proliferation and fibrogenesis of hepatic stellate cells by inhibition of Smad2/3 phosphorylation. Biomed Pharmacother. 2018;107:1728-35. 
30. Ge PS, Runyon BA. Treatment of Patients with Cirrhosis. N Engl J Med. 2016;375:2104-5.

31. Chang ML, Yeh CT, Chang PY, Chen JC. Comparison of murine cirrhosis models induced by hepatotoxin administration and common bile duct ligation. World $\mathrm{J}$ Gastroenterol. 2005;11:4167-72.

32. Zhang Z, Lin H, Shi M, Xu R, Fu J, Lv J, et al. Human umbilical cord mesenchymal stem cells improve liver function and ascites in decompensated liver cirrhosis patients. J Gastroenterol Hepatol. 2012;27:112-20.

33. Harrington K, Alvarez-Vallina L, Crittenden M, Gough M, Chong H, Diaz RM, et al. Cells as vehicles for cancer gene therapy: the missing link between targeted vectors and systemic delivery? Hum Gene Ther. 2002;13:1263-80.

34. Merrell AJ, Stanger BZ. Adult cell plasticity in vivo: de-differentiation and transdifferentiation are back in style. Nat Rev Mol Cell Biol. 2016;17:413-25.

35. Karp JM, Leng Teo GS. Mesenchymal stem cell homing: the devil is in the details. Cell Stem Cell. 2009;4:206-16.

36. Yu Y, Wu RX, Gao LN, Xia Y, Tang HN, Chen FM. Stromal cell-derived factor-1directed bone marrow mesenchymal stem cell migration in response to inflammatory and/or hypoxic stimuli. Cell Adh Migr. 2016;10:342-59.

37. Sakaida I, Terai S, Yamamoto N, Aoyama K, Ishikawa T, Nishina H, et al. Transplantation of bone marrow cells reduces $\mathrm{CCl} 4$-induced liver fibrosis in mice. Hepatology.2004;40:1304-11.

38. Chen J, Li Y, Wang L, Zhang Z, Lu D, Lu M, et al. Therapeutic benefit of intravenous administration of bone marrow stromal cells after cerebral ischemia in rats. Stroke.2001:32:1005-11.

39. Shi D, Xin J, Lu Y, Ding W, Jiang J, Zhou Q, et al. Transcriptome profiling reveals distinct phenotype of human bone marrow mesenchymal stem cell-derived hepatocyte-like cells. Int J Med Sci. 2020;17:263-73.

40. Coronado RE, Somaraki-Cormier M, Ong JL, Halff GA. Hepatocyte-like cells derived from human amniotic epithelial, bone marrow, and adipose stromal cells display enhanced functionality when cultured on decellularized liver substrate. Stem Cell Res. 2019;38:101471.

41. Mou XZ, Lin J, Chen JY, Li YF, Wu XX, Xiang BY, et al. Menstrual blood-derived mesenchymal stem cells differentiate into functional hepatocyte-like cells. J Zhejiang Univ Sci B. 2013;14:961-72.

42. Ke Z, Zhou F, Wang L, Chen S, Liu F, Fan X, et al. Down-regulation of Wnt signaling could promote bone marrow-derived mesenchymal stem cells to differentiate into hepatocytes. Biochem Biophys Res Commun. 2008;367:342-8.

43. Milhem M, Mahmud N, Lavelle D, Araki H, DeSimone J, Saunthararajah $Y$, et al. Modification of hematopoietic stem cell fate by 5aza 2'deoxycytidine and trichostatin A. Blood.2004;103:4102-10.

44. Sakaguchi $T$, Kono $Y$, Itaba N, Morimoto M, Isomoto $H$, Shiota G. Identification of a novel deactivating small-molecule compound for fibrogenic hepatic stellate cells. Yonago Acta Med. 2020;63:79-87.

45. Yu F, Ji S, Su L, Wan L, Zhang S, Dai C, et al. Adipose-derived mesenchymal stem cells inhibit activation of hepatic stellate cells in vitro and ameliorate rat liver fibrosis in vivo. J Formos Med Assoc. 2015;114:130-8.

46. Yang X, Meng Y, Han Z, Ye F, Wei L, Zong C. Mesenchymal stem cell therapy for liver disease: full of chances and challenges. Cell Biosci. 2020;10:123.

47. Berardis S, Dwisthi Sattwika P, Najimi M, Sokal E. Use of mesenchymal stem cells to treat liver fibrosis: current situation and future prospects. World J Gastroenterol. 2015:21:742-58.

48. Lee C, Kim M, Han J, Yoon M, Jung Y. Mesenchymal stem cells influence activation of hepatic stellate cells, and constitute a promising therapy for liver fibrosis. Biomedicines. 2021;9:1598.

49. Qu Y, Zong L, Xu M, Dong Y, Lu L. Effects of 18alpha-glycyrrhizin on TGF-beta1/ Smad signaling pathway in rats with carbon tetrachloride-induced liver fibrosis. Int J Clin Exp Pathol. 2015;8:1292-301.

50. Fang L, Huang C, Meng X, Wu B, Ma T, Liu X, et al. TGF-beta1-elevated TRPM7 channel regulates collagen expression in hepatic stellate cells via TGF-beta1/ Smad pathway. Toxicol Appl Pharm. 2014;280:335-44.

51. Zhang L, Zhou D, Li J, Yan X, Zhu J, Xiao P, et al. Effects of bone marrow-derived mesenchymal stem cells on hypoxia and the transforming growth factor beta 1 (TGFbeta-1) and SMADs pathway in a mouse model of cirrhosis. Med Sci Monit. 2019;25:7182-90.
52. Henderson NC, Arnold TD, Katamura Y, Giacomini MM, Rodriguez JD, McCarty JH, et al. Targeting of alphav integrin identifies a core molecular pathway that regulates fibrosis in several organs. Nat Med. 2013;19:1617-24.

53. Reed $\mathrm{NI}$, Jo $\mathrm{H}$, Chen $\mathrm{C}$, Tsujino $\mathrm{K}$, Arnold TD, DeGrado WF, et al. The alphavbeta1 integrin plays a critical in vivo role in tissue fibrosis. Sci Transl Med. 2015;7:288ra79.

54. Su P, Chen S, Zheng YH, Zhou HY, Yan $\mathrm{CH}_{1} \mathrm{Yu}$ F, et al. Novel function of extracellular matrix protein 1 in suppressing Th17 cell development in experimental autoimmune encephalomyelitis. J Immunol. 2016;197:1054-64.

\section{ACKNOWLEDGEMENTS}

We thank to all the peer reviewers and editors for their opinions and suggestions.

\section{AUTHOR CONTRIBUTIONS}

SZJ and QL designed the study. QL contributed to the cytology experiments, animal experiments, data acquisition, data analysis, and manuscript writing. CQL, $\mathrm{QQH}$ and LZ provided experimental technical support. XLS, DDN, JYL, and YNJ participated in the statistical analysis of the data. The authors read and approved the final manuscript.

\section{FUNDING}

This study was supported by the Science Funding for Distinguished Younger Scholars from the Second Affiliated Hospital of Harbin Medical University.

\section{COMPETING INTERESTS}

The authors declare no competing interests.

\section{ETHICS APPROVAL AND CONSENT TO PARTICIPATE}

All experiments were conducted in accordance with the guidelines and the experimental protocol for animals was approved by the Experimental Center of the Second Affiliated Hospital of Harbin Medical University (No. SYDW2019-240).

\section{ADDITIONAL INFORMATION}

Correspondence and requests for materials should be addressed to Shizhu Jin.

Reprints and permission information is available at http://www.nature.com/ reprints

Publisher's note Springer Nature remains neutral with regard to jurisdictional claims in published maps and institutional affiliations.

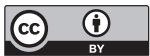

Open Access This article is licensed under a Creative Commons Attribution 4.0 International License, which permits use, sharing, adaptation, distribution and reproduction in any medium or format, as long as you give appropriate credit to the original author(s) and the source, provide a link to the Creative Commons license, and indicate if changes were made. The images or other third party material in this article are included in the article's Creative Commons license, unless indicated otherwise in a credit line to the material. If material is not included in the article's Creative Commons license and your intended use is not permitted by statutory regulation or exceeds the permitted use, you will need to obtain permission directly from the copyright holder. To view a copy of this license, visit http://creativecommons. org/licenses/by/4.0/.

(c) The Author(s) 2022 